\title{
EL IMPACTO DE LA RENOVACIÓN CARISMÁTICA EN LA IGLESIA CATÓLICA DE GUATEMALA
}

Jakob Egeris Thorsen

Recibido: 17/02/2016 Aceptado: 28/04/2016

\begin{abstract}
Resumen
Después de una introducción histórica al movimiento de la Renovación Carismática Católica (RCC) en América Latina, este artículo describe etnográficamente cómo el catolicismo carismático se desarrolla hoy en día en Guatemala, y cómo afecta la vida diaria de una parroquia católica en la Ciudad de Guatemala. Analiza el impacto de la RCC en los documentos de la Iglesia institucional y en la implementación de diversos planes pastorales a nivel nacional y latinoamericano. El artículo está basado principalmente en un trabajo de campo (junio-diciembre de 2009) en la Ciudad de Guatemala, más uno (de octubre de 2014 a enero de 2015) en Santiago Atitlán (Sololá, Guatemala). ${ }^{1}$
\end{abstract}

Palabras clave: Renovación Carismática Católica (RCC); Guatemala; pentecostalización; CELAM; catolicismo; movimientos eclesiales.

\begin{abstract}
After a short historical introduction to The Catholic Charismatic Renewal (CCR) in Latin America, this article provides an ethnographic description of how the lay movement has developed in Guatemala and how it affects the daily religious life of a parish in Guatemala City. It furthermore analyzes the impact of the CCR on the institutional Church, its official documents, and on pastoral plans and their implementation on a Guatemalan and Latin American level. The ethnographic descriptions are based on a six-month fieldwork in Guatemala City (June to December 2009) and a three-month fieldwork in Santiago Atitlán (October 2014 to January 2015).
\end{abstract}

Key words: Catholic Charismatic Renewal (CCR); Guatemala; Pentecostalization; CELAM; Catholicism; Ecclesial movements.

\section{Introducción}

La Renovación Carismática surgió en la Iglesia católica en el año 1967. Formaba parte de la llamada "segunda ola" que desde el final de la década de 1950 llevó al avivamiento pentecostal a las iglesias históricas. En el caso del catolicismo, tal apertura a nuevas experiencias y formas de expresión de la fe se hizo posible hasta después del Segundo Concilio Vaticano (1962-1965), y surgió en medio del entusiasmo, 
la confusión y la curiosidad producidos en años posteriores. En febrero de 1967, varios miembros de la facultad y estudiantes de la Universidad de Duquesne, institución católica ubicada en Pittsburgh (EE. UU.), experimentaron una "efusión del Espíritu", con el "bautismo del Espíritu Santo" acompañado del "don de hablar en lenguas". El movimiento creció rápidamente y los grupos de oración carismáticos se extendieron a otras universidades católicas y, en último lugar, a las parroquias católicas en los Estados Unidos. Al respecto, Edward Cleary describió que un evento similar tuvo lugar en Bogotá (Colombia) ese mismo año. Después de llamarse, en un inicio, "católicos pentecostales", el movimiento empezó a nombrarse "renovación carismática católica", indicando la supuesta misión de restaurar los carismas de la iglesia primitiva que habían quedado obsoletos. El origen de la RCC, por lo tanto, parece tener más de un origen, aunque Duquesne es oficialmente reconocido y celebrado como el origen por la misma organización (Cleary, 2011).

La propagación de la RCC a los diferentes países de América Latina en el comienzo de la década de 1970 siguió un patrón: fue introducido generalmente por sacerdotes de América del Norte, quienes, por invitación de obispos o sacerdotes locales interesados, organizaron retiros llamados "Vida en el Espíritu". Aquí los participantes fueron introducidos a los signos carismáticos, como el hablar en lenguas, oración por la sanación y la experiencia del bautismo en el Espíritu Santo. Después de su iniciación, sacerdotes y hermanas religiosas extendieron el movimiento a las instituciones educativas católicas y de allí a las parroquias (Chesnut, 2003). Una figura prominente de la propagación fue el entonces fraile dominico Francis MacNutt, quien independientemente del evento Duquesne había experimentado el bautismo del Espíritu durante un retiro protestante carismático en 1967. MacNutt, sobre todo, se ha dedicado a la oración por los enfermos y para sanación (MacNutt, 1974; MacNutt, 1995; MacNutt, 2005). Además, fue un misionero incansable de la RCC y un iniciador importante del movimiento. Viajó a Bolivia y a Perú en 1970, a República Dominicana en 1971 y a Guatemala, México, Costa Rica, Colombia y Chile en 1972, y a Guatemala en 1972 (Cleary, 2011).

La RCC experimentó una fuerte oposición desde varias aristas. Por un lado, algunos obispos conservadores tenían dudas acerca de la catolicidad del movimiento; pues, como la manera de expresión religiosa se parecía a las crecientes iglesias pentecostales, se preguntaban si estaban permitiendo un movimiento de espíritu protestante en el seno de la Iglesia, el cual, con el tiempo, podría funcionar como una transición al protestantismo para los fieles católicos (Chesnut, 2003). Por otro lado, la oposición provino de obispos y teólogos inspirados de la teología de la liberación, quienes en la RCC vieron reducida la fe a lo puramente espiritual, con cual la Iglesia traicionaría su compromiso con la justicia social y su opción preferencial por los pobres (Cleary, 2007).

A pesar de la oposición de varios sectores de la Iglesia, la RCC se extendió rápidamente. Durante la década de 1980, creció hasta convertirse en el mayor movimiento 
laico en Latinoamérica, y también fue cuando se integró más en la institución eclesial. Una vez establecida en la mayoría de las diócesis, su relación con los obispos se formalizó con la instrucción de sacerdotes supervisores y el establecimiento de organizaciones locales y nacionales de la RCC. En cuanto al número de RCC-participantes de América Latina, una estimación calificada del año 2000 señala que había 74 millones de carismáticos católicos (de una población de 520 millones en Latinoamérica el mismo año). Este número se deriva de la enciclopedia World Christian Trends de Barrett \& Co. y se basa en una encuesta llevado a cabo durante cuatro años por la oficina central de la RCC (ICCRS) con sede en Roma (Barrett y Co., 2001; Cleary, 2011). En este análisis, Colombia y Brasil aparecen como las naciones con mayor porcentaje de carismáticos católicos, 28 y 22 por ciento, respectivamente, mientras que países como Nicaragua y Paraguay tienen el número más bajo del continente con menos de cinco por ciento. De acuerdo con esta estadística, el 9 por ciento de los católicos guatemaltecos son carismáticos, pero este número es muy controvertido, como se verá más adelante.

\section{La RCC en Guatemala}

Durante una visita a EE. UU., el arzobispo de Ciudad de Guatemala, Mario Cardenal Casariego († 1983), invitó personalmente al padre Harold Cohen SJ para que llevara a cabo retiros de la Renovación Carismática Católica en la Ciudad de Guatemala en diciembre 1973. Los retiros del padre Cohen representan el nacimiento oficial de la RCC en Guatemala. Sin embargo, el año anterior dos hermanas religiosas del orden Maryknoll habían organizado retiros carismáticos, y en septiembre del 1973 tres hermanas religiosas y un laico participaron en uno dirigido por Francis MacNutt (Cleary, 2011). A mediados de la década, MacNutt y Ruth Stapleton organizaron otro retiro donde 35 líderes laicos recibieron el bautismo en el Espíritu Santo (Chesnut, 2003; Early, 2012).

Durante los años setenta, tanto la Iglesia como el país estaban en crisis. La guerra civil entre los gobiernos militarizados y las fuerzas guerrilleras se estaba intensificando, y en la Iglesia había continuas disputas sobre la línea pastoral después del Segundo Concilio Vaticano y su recepción latinoamericana en la Segunda Conferencia del CELAM en Medellín (1968). Además, la teología de la liberación había entrado en Guatemala, especialmente a través de los sacerdotes y las religiosas extranjeras de quienes la Iglesia de ese país dependía fuertemente. Al contrario de muchas otras naciones de América Latina, donde la RCC entró por "la puerta trasera" y a menudo enfrento cierta resistencia por el episcopado, sI fue invitada a la Arquidiócesis de Guatemala, e ingresó por la entrada principal del palacio arquidiocesano. Durante el retiro del padre Cohen, el obispo auxiliar Mons. Ramiro Pellecer recibió el bautismo en el Espíritu y luego se volvió un ancla para el movimiento en la capital. En 1974 se establecieron grupos de oración en varios colegios católicos como el Instituto Belga 
Guatemalteco. Desde estos puntos de partida, los grupos de oración de la RCC comenzaron a crecer y difundirse en el área metropolitana. ${ }^{2}$

Mientras que las relaciones entre la RCC y la Iglesia institucional se desarrollaban relativamente sin problemas en la Ciudad de Guatemala, este no siempre fue el caso en el interior del país, donde la RCC causó polémica y divisiones en muchas diócesis y parroquias. En la RCC fue introducida y se difundía de forma gradual en las áreas rurales por medio de sacerdotes y religiosas estadounidenses que vivieron la experiencia carismática en la capital o en el extranjero, o bien por líderes laicos que se convirtieron en carismáticos (Early, 2012; Gutiérrez, 2014).

En muchos lugares, la llegada de la RCC causó conflictos con los laicos de la Acción Católica, incluso fue prohibida por los párrocos o el obispo. Esto causó divisiones en varias parroquias y en numerosos casos dio como resultado las conversiones colectivas de grupos carismáticos a iglesias pentecostales. La razón de la oposición contra el movimiento de la RCC se debió a la sospecha de los clérigos y laicos comprometidos de que se orientaba exclusivamente hacia la piedad personal y la experiencia religiosa, razón por la cual traicionaría la orientación social de la Iglesia; perseguida durante los años del culmen de la guerra civil 1979-1983 por esa razón (Falla, 2001). Cuando el ejército nacional expulso a el clero católico y a las religiosas de las zonas de conflicto, la Renovación Carismática era el único movimiento católico que podía continuar sus reuniones y actos de celebración (Stoll, 1993; Melander, 1999).

Hay numerosos católicos carismáticos entre las víctimas de la violencia de la época. A pesar de eso, era más seguro pertenecer a la RCC que a otros grupos católicos. A diferencia de los sacerdotes, las hermanas religiosas y el laicado organizado en la Acción Católica, la RCC en general no fue percibida como un "problema insurgente" por los militares, ya que no se dedicaba a la promoción de derechos humanos o al apoyo de organizaciones campesinas. Por esta razón, los grupos carismáticos (junto con las iglesias evangélicas) llegaron a ser un refugio para muchas personas cuando todas las demás estructuras de la Iglesia católica se habían desmontado (Melander, 1999; Falla, 2001; Early, 2012). En áreas rurales de Guatemala, los carismáticos católicos muchas veces construyeron una casa de culto aparte de la iglesia parroquial $\mathrm{y}$, en muchos en lugares, la parroquia se dividió entre la mayoría Acción Católica y el grupo carismático. Aunque con el paso del tiempo estas divisiones han desaparecido cada vez más, todavía persisten en algunas áreas (Althoff, 2005; Hoenes, 2011). La diócesis de Huehuetenango es un ejemplo especial, pues los sacerdotes misioneros de Maryknoll permitieron que la RCC se estableciera allí; hasta que fue prohibida por el obispo Rodolfo Bobadilla Mata. Hasta su jubilación en el año 2012, fue la única diócesis, donde por décadas no se permitió la RCC. Esto obligó a los católicos carismáticos de Huehuetenango a organizarse de forma independiente de la RCC y de una manera semiclandestina, ya que los sacerdotes de muchas parroquias sancionaron la pertenencia a grupos de oración carismáticos con la negación de los sacramentos. 
Resulta difícil obtener información de por qué la situación se había desarrollado de esta manera, pero al parecer el antagonismo entre diócesis y carismáticos se desarrolló hasta un punto en el cual el diálogo no era posible. En la diócesis de Sololá-Chimaltenango, por ejemplo, un conflicto entre la Acción Católica, la RCC, las cofradías y la Iglesia institucional resultó en un cismo eclesial en donde parte de la feligresía siguió al padre excomulgado, Eduardo Aguirre, a una nueva "Iglesia Católica Ecuménica Renovada", que a su vez forma parte de la "Iglesia Católica Apostólica Siro-Ortodoxa de Antioquia". Muchos otros grupos carismáticos en el altiplano de Guatemala se han incorporado a esta.

En la capital, por su parte, la RCC ha disfrutado de un amplio apoyo. El Obispo auxiliar Mons. Ramiro Pellecer (nacido 1929 y jubilado en el 2010) experimentó el bautismo en el Espíritu Santo durante el primer retiro del jesuita Cohen en 1973, momento en el cual se convirtió en el ancla institucional del movimiento en la arquidiócesis. Asimismo, el obispo auxiliar Rodolfo Mendoza (nacido en 1934), el padre René Estrada y su hermano, el asesor nacional de la RCC, padre Hugo Estrada, fueron algunos de los primeros participantes, asegurando el apoyo al movimiento entre el clero nacional concentrado en la región capitalina. En el área rural el apoyo del clero extranjero era mucho más disperso.

Debido a los desacuerdos sobre de la línea pastoral frente la RCC hasta el 1986, los obispos de Guatemala lograron una postura colectiva. Con la carta pastoral "Renovados en el Espíritu" se aprobó el movimiento oficialmente, pero este hecho se vio acompañado de muchas advertencias. Aunque la instrucción elogia al movimiento por su capacidad para involucrar y comprometer a los laicos, también advierte de una serie de riesgos, entre ellos el fundamentalismo en lectura bíblica y un sentimentalismo excesivo (CEG, 1986).

\section{Extensión carismática en Guatemala}

De acuerdo con la enciclopedia World Christian Trends, el porcentaje de católicos guatemaltecos vinculados con la RCC es de un $9 \%$, lo que sitúa al país muy por detrás de otras naciones como Colombia (28 \%) y Brasil (22 \%) (Barrett \& Co. 2001). Según Edward Cleary, quien fue observador del movimiento durante décadas, los números de Barrett \& Co. son los más fiables, pues se basan en censos locales por la RCC, que se han comparado con la encuestas públicas (Cleary, 2011). Sin embargo, existen números muy diferentes, por ejemplo en el informe del Pew Forum sobre el cristianismo pentecostal y carismático del año 2006 se da la sorprendente cifra de que el $62 \%$ de los católicos guatemaltecos está categorizado como carismáticos, en comparación con el 57 \% de los católicos brasileños (Pew Forum, 2006). En otro informe del año 2014 sobre la religión en América Latina, el $38 \%$ de los católicos guatemaltecos se identifica como "carismático" (Pew Forum, 2014). 
Otras fuentes indican lo mismo; por ejemplo, en el libro The Polictics of the Spirit del 2001, Timothy Steigenga ha realizado encuestas sobre el predominio de los elementos carismáticos y pentecostales en la vida religiosa de guatemaltecos en los departamentos de la Ciudad de Guatemala y de la vecina Sacatepéquez. Aquí se demuestra que el 71,6 \% de los católicos había experimentado una "conversión personal", el 70,6\% fue testigo de una sanación milagrosa, el 63,4 \% se vio "tentado por el diablo" y el 19,8\% indicaba haber hablado en lenguas (Steigenga, 2001). Los últimos números que deben mencionar antes de pasar a una discusión sobre cómo entender estos resultados divergentes, es un censo del 2008 realizado por la RCC en Guatemala. Aunque faltaban los números de cuatro departamentos rurales, la encuesta indica que en el 2008 a nivel nacional existían 1044 grupos de oración de la RCC, 89835 miembros registrados y 16782 "servidores" comprometidos (CCR Guatemala, 2008). Estas figuras de carismáticos organizados por la RCC corresponde solo a un 1,5\% de los católicos guatemaltecos, aproximadamente (si se asume que alrededor de 9 millones de los 14 millones de habitantes de Guatemala profesan este credo). ¿Cómo dar sentido a esos números?

Es importante distinguir entre los carismáticos organizados en la RCC que se autodefinen claramente como carismáticos, y aquellos que forman parte de los grupos de oración independientes y no tienen ninguna conexión directa con la RCC. Existen grupos parroquiales y grupos más independientes. Algunos de estos son el resultado de la aplicación de programas de evangelización pastoral como el Sistema Integral de la Nueva Evangelización (SINE), el cual creó el sacerdote mexicano Padre Alfonso Navarro y fue implementado en muchas diócesis, especialmente en México y Centroamérica en la última parte de la década de los noventa. Sin embargo, diez años después había sido abandonado en la mayoría de los lugares.

La idea central del SINE era que todos los grupos de oración debían estar basados en la parroquia y no tener relación con los movimientos nacionales o internacionales, como la RCC. La implementación del SINE creó un montón de conflictos y también una gran cantidad de grupos de oración carismática independientes, que, no aparecen en las estadísticas de la RCC. Aparte del SINE, diversas prácticas carismáticas se han difundido a otros grupos de oración de laicos a lo largo de los últimos cuarenta años, lo cual resulta en el hecho estadísticamente inquietante de que el catolicismo carismático en Guatemala va más allá de la RCC.

Ante esto, si se analiza la situación, y se toma en cuenta que las categorías utilizadas para definir a los carismáticos son fluidas y debatidas, razón por la cual resulta necesario distinguir entre dos tipos de carismáticos católicos: los "completos" y los "semicarismáticos". Los primeros son los miembros clásicos de la RCC, que han pasado por una experiencia de conversión fuerte combinada con el fenómeno del bautismo en el Espíritu Santo. Además, poseen una fuerte identidad como "carismáticos", están unidos firmemente a sus grupos de oración 
y comunidades y practican la gama completa de los carismas: hablar en lenguas, el bautismo en el Espíritu Santo, el descanso en el Espíritu y la oración por sanación con imposición de manos.

Por su parte, el otro grupo, los "semicarismáticos", ha adoptado la mayoría de los elementos carismáticos menos espectaculares, como las alabanzas carismáticas centradas en el Espíritu Santo (que en su mayoría fueron tomadas de las iglesias pentecostales), la "predicación bíblica" dirigida a la conversión personal, la restauración moral, el uso de testimonios personales y el intenso estilo de la oración libre. Muchos grupos de oración independientes son "semi-" o en algún punto intermedio en la escala entre "completos" y "semi-".

A partir de esto, surge la interrogante de ¿cómo se puede utilizar esta segmentación para aclarar las estadísticas divergentes mencionadas anteriormente? En primer lugar, podría utilizarse para entender por qué existe tanta variedad. Si un investigador le pregunta a la gente si ha experimentado la oración de sanación, una conversión profunda, el hablar en lenguas o ha sido tentado por el diablo, etc., como lo hizo Steigenga (2001) o el informe del Pew Forum (2006), obtendrá un alto porcentaje de "carismáticos católicos". Sin embargo, probablemente la mayoría de estas personas no forma parte activa de un grupo de la RCC. Algunos de ellos participan en otros grupos laicos con tinte carismático suave, mientras otros, quizás, han experimentado una conversión o una sanación durante un curso o retiro prebautismal o prematrimonial, mientras que algunos pueden haber buscado a un grupo de oración carismático para la sanación durante un período de enfermedad o angustia emocional para luego dejar de asistir.

En la encuesta publicada por el Pew Forum en el 2014, el 38 por ciento de los católicos en Guatemala respondió afirmativamente a la siguiente pregunta: “Usted se describiría como un(a) carismático(a) o no?" (Pew Forum, 2014: 64, 184). Si solo cuenta a los participantes de los grupos de oración de la RCC se obtendrá un número significativamente menor. El problema central es que los elementos carismáticos han permeado a otros grupos católicos en Guatemala; por esta razón se hace cada vez más difícil definir claramente quiénes son o no en realidad "carismáticos".

En Guatemala, a partir de la investigación realizada para este artículo, entre el 30 y 40 por ciento de los católicos bautizados se puede calificar como semicarismático y de 5 a 10 por ciento como carismático completo. Esta evaluación se basa en las existentes estadísticas incompletas, en la observación personal y en entrevistas con los obispos Mons. Mendoza y Mons. Pellecer y con la coordinadora nacional de la RCC María Eugenia de Góngora. Así, la investigación estima el número de semicarimáticos más bajo que el informe de Pew Forum de 2006, pues no todo lo que el informe califica como "carismático" ni necesariamente se pueda vincular con la tradición pentecostalcarismática (por ejemplo, haber sido "testigo de un milagro" o haber "experimentado espíritus malignos" (Pew Forum, 2006: 140-143). Más bien, la evaluación se corresponde con el informe del Pew Forum del 2014, donde se indicó un porcentaje de 38 por ciento 
carismáticos entre los católicos guatemaltecos (Pew Forum, 2014). Por lo tanto, el estudio del número de "carismáticos completos" está más vinculado con los números de Barrett \& Co., y es más alto que el censo no publicado por la RCC en Guatemala (CCR Gua., 2008).

Debido a que muchos católicos bautizados en Guatemala son practicantes ocasionales, los católicos carismáticos ("completos" y "semi-") representan un mayor porcentaje de los que practican la religión con regularidad. También constituyen una proporción significativa de los católicos que tienen algún cargo en la Iglesia, por ejemplo como catequistas, delegados de la Palabra, ministros extraordinarios de la comunión y lectores.

\section{Una parroquia renovada}

La parroquia Santísima Trinidad está ubicada en las afueras de la capital. ${ }^{3}$ Para el año 2009 en la colonia residencial vivían cerca de 10000 personas, de las cuales aproximadamente dos terceras partes eran católicos, y una tercera parte pertenecía a una de las varias iglesias protestantes. La mayoría de los residentes locales se puede describir como de clase media baja. Las casas son modestas, los carros viejos. Al mismo tiempo, esta colonia está rodeada por barrios más pobres, ubicados en la periferia de la ciudad y en las laderas de los barrancos que cortan a través del valle capitalino. El corazón de la colonia es su iglesia parroquial, un edificio amplio y funcional de la década de los noventa, construido en sustitución de la antigua iglesia. La nueva edificación tiene capacidad para unas 400 personas, y el domingo el párroco celebra tres misas a las que asisten gran cantidad de feligreses. Para los actos litúrgicos de martes a sábado llegan alrededor de cincuenta personas. En la iglesia también hay un salón parroquial donde muchos de los grupos de laicos (de 25 a 100 participantes) se congregan durante la semana. Además, unos 15 o 20 pequeños grupos de estudio bíblico se reúnen en casas privadas. Muchas de estas agrupaciones iniciaron sus actividades en la década de los setenta como comunidades eclesi ales de base (CEB), pero por las controversias que rodeaban a la teología de la liberación en los años ochenta se les llamó desde entonces "pequeñas comunidades eclesiales". En el 2009, la mayoría de los grupos que se juntaban en el salón tenían un perfil carismático, a pesar de que ninguno estaba organizado como parte de la RCC.

El grupo llamado "Espíritu de Dios" fue dirigido exclusivamente por doña Concha, quien entonces tenía 66 años y estuvo a cargo del grupo durante 26 años. Ella creció como hija única en una cabecera departamental en una familia ladina de la clase media baja. A los doce años su padre abandonó el hogar y se fue a vivir a otro departamento con otra mujer. La situación económica se volvió precaria, por lo que madre, hija y abuela (quien vivía con ellas) pasaron muchas penas. Era un hogar católico, asistían a misa varias veces por semana y en casa rezaban el rosario. 
De niña, doña Concha soñaba con convertirse en monja; a los 16 años solicitó el ingreso pero la madre superiora se lo negó porque venía de un hogar desintegrado, lo cual le generó una gran decepción. Con un dinero heredado, la mamá logró comprar una pequeña casa en la capital a donde fueron a vivir para que doña Concha estudiara enfermería, profesión a cual se dedicó toda su vida laboral. A principios de los años setenta se casó con un médico y tuvieron una hija.

En 1976, año del gran terremoto, su esposo la abandonó por una mujer más joven y poco tiempo después a su hija se le diagnosticó una enfermedad grave. Doña Concha cayó en una depresión muy grave. No podía trabajar y su mamá tuvo que hacerse cargo del hogar y de su hija enferma. Aunque nunca faltó a sus oraciones, esto no le daba consuelo. Inclusive pensó en suicidarse. Fue entonces cuando apareció un conocido que la llevó a un grupo de oración de la renovación carismática. Después de varias visitas al grupo experimentó sanación, empezó a hablar en lenguas y vivió el bautismo en el Espíritu Santo. Unos años más tarde se dio cuenta de que tenía "el don de la sanación" y comenzó a orar por las personas con imposición de manos. Entonces, ahora vivía en la colonia donde participaba en una comunidad eclesial de base, que luego se transformó en la comunidad carismática del "Espíritu de Dios”, dirigida por ella.

Como dirigente, tenía a su lado un grupo de veinte servidores aproximadamente, quienes la ayudaban a organizar las reuniones semanales, las cuales eran el núcleo de la labor del grupo. Además de los servidores llegaban alrededor de 40 personas cada semana, la mayoría mujeres. Las reuniones comenzaban siempre con una media hora de alabanzas dirigidas por una pequeña banda musical. Las melodías eran alegres y recurrían a populares. Las letras fueron siempre muy centradas en el Espíritu Santo y muchas veces servían como una invocación del mismo; en su mayoría eran las mismas que se cantaban en las iglesias pentecostales. Después de las alabanzas, que solo se habían interrumpido por breves oraciones, se hacía una devoción a la Virgen María, seguida de la predica del día.

En la Ciudad de Guatemala, el predicador suele ser un laico asociado con uno de los muchos "ministerios de predicación" que envían predicadores a los cientos de grupos de oración de las parroquias. Ellos solo pueden ser invitados con el permiso del párroco. En el grupo del "Espíritu Santo", así como en muchos grupos, el predicador antes de iniciar la prédica se arrodillaba delante de los congregados y toda la gente extendía sus manos hacia él y acompañaban a doña Concha para pedir la bendición de Dios. La prédica luego se basaba en uno de los textos de la liturgia del domingo siguiente. El punto de partida en la mayoría de las prédicas era sobre la vida cotidiana de los presentes, sus problemas y desafíos, en esta el predicador trataba de hacer sugerencias específicas sobre cómo actuar desde la luz de la fe, por ejemplo sobre cómo pedir la ayuda de Dios para resolver los problemas en la familia, en la pareja y en el trabajo. Aunque siempre se destacaba la necesidad de arrepentirse y convertirse para realizar el cambio moral, las sugerencias en las predicas tenían un carácter 
práctico, como controlar el temperamento, dejar de beber, evitar peleas, etc. Después del sermón, la pequeña banda tocaba de nuevo, ahora más lento, e interpretaban alabanzas sentimentales que preparaban a la gente para las oraciones de intercesión y sanación, para esto llevaban a los niños fuera del recinto bajo supervisión de una de las ayudantes, y luego cerraban las puertas. Doña Concha invitaba a las personas que padecían enfermedades, traumas psicológicos y depresión a pasar adelante y colocarse de rodillas. Mientras tanto, la banda seguía tocando de fondo. El ambiente se percibía muy intenso. Doña Concha caminaba despacio a lo largo de la fila de arrodillados imponiendo las manos sobre las cabezas y orando intensamente por las personas. Ella les ordenó a los demonios salir de aquellos cuerpos, habló en lenguas y abrazó a las personas, quienes a menudo terminaban cayéndose al suelo en un estado de trance, llamado "descanso del Espíritu". Debido a que muchas de estas personas eran frágiles y mayores, los servidores se ubicaban detrás de ellas, listos para sostenerlas cuando cayeran. A veces espasmos sacudieron a las personas y en otras ocasiones empezaban a vomitar. En esos casos se traía papel periódico para secar el suelo, y agua y servilletas para quienes se vieran afectados. Después de la sanación entonaban unas alabanzas alegres de agradecimiento y doña Concha terminaba con una oración. Luego había siempre un café y un pan dulce. Los domingos, los miembros del grupo de oración "Espíritu de Dios" iban a misa junto con los demás feligreses y muchos de los servidores también tenían cargos en la misa como ministros extraordinarios de la comunión o como ayudantes de asuntos prácticos (limpieza, etc.).

Aquí no hay espacio para presentar a los servidores y a los participantes regulares, que en el caso de “Espíritu de Dios" en su mayoría eran mujeres, pues -al igual que doña Concha- habían experimentado problemas de pareja, sufrimiento nervioso por abusos en la niñez/juventud o enfermedades graves, lo cual las había llevado a participar en el grupo. El caso de los hombres que integraban otros grupos era diferente, ya que según lo documentado en las autobiografías, ellos vivieron abusos en el consumo del alcohol y problemas familiares y conyugales concomitantes. Aquí la participación en el grupo carismático pasa alentado por la pareja o la familia. Al igual que en las iglesias evangélicas, este grupo religioso provee al hombre de una comunidad masculina alternativa, que le facilita abandonar el vicio, "la vida en la calle" y responsabilizarse de su familia y hogar (O’Neill, 2010; Chesnut, 2003; Cleary, 2011 y Theije, 1998).

Este patrón de conversión masculino a la RCC se encontró tanto entre hombres ladinos de la clase media baja en la capital, como en hombres indígenas comerciantes en Santiago Atitlán (trabajo de campo 2014-2015). Sin embargo, el caso es diferente con el grupo juvenil (de 15 a 30 años) del 2009. Los participantes eran de clase media-alta, procedentes de familias católicas practicantes y la mayoría no había tomado alcohol con ninguna frecuencia. En esos casos la participación en el grupo tenía el objetivo de una búsqueda de identidad y de desarrollo personal. 
El grupo “Espíritu de Dios” descrito arriba es un ejemplo de cómo se vive la renovación carismática en Guatemala, pues constituye una parroquia donde hay una variedad de grupos laicos, la gran mayoría carismáticos. También, es un grupo sin ningún vínculo oficial con la organización de la RCC y así un ejemplo de las dificultades de estadística presentes en ese campo. En Guatemala existen miles de grupos de oración de estilo carismático y a nivel latinoamericano han de llegar a los cientos de miles. Quizás lo anterior se debe a que estos grupos han ganado una gran influencia tanto en la vida diaria de las parroquias como en el nivel del liderazgo de la Iglesia, el episcopado.

\section{El impacto de la RCC en la Iglesia católica a nivel continental}

La Conferencia Episcopal de América Latina (CELAM) celebró su quinta conferencia general en Aparecida (Brasil) en mayo del 2007. En dicha sesión se analizó la situación de la Iglesia católica y se trató de formular una estrategia pastoral de conjunto para los próximos diez y veinte años. En el documento y mensaje finales, los obispos anunciaron a una nueva "misión continental", una campaña de avivamiento que se aplicará en todas las diócesis desde México hasta el Cono Sur. La misión continental es un nuevo esfuerzo para revitalizar la "nueva evangelización" que se había anunciado durante la cuarta conferencia general (en Santo Domingo, 1992), pues los procesos de transformación de la sociedad y de campo religioso que han caracterizado a Latinoamérica durante los últimos 70 años se han acelerado desde 1992. América Latina, que tradicionalmente se llamaba "el continente católico", es cada vez más pluralista y el monopolio religioso se ha roto; en primer lugar, porque del 15 al 20 por ciento de los latinoamericanos ha dejado de profesar esta religión y se convirtieron al protestantismo, en su gran mayoría de perfil (neo) pentecostal y evangélico (Cleary, 2004; Barrett \& Co., 2001).

En el Documento de Aparecida, los obispos retoman el mismo procedimiento como en las conferencias generales de Medellín (1968) y Puebla (1979). El texto final se estructura en los siguientes tres pasos: ver, juzgar y actuar. Como los nombres de los pasos sugieren, la primera parte del documento consiste en una descripción de la realidad religiosa, social y cultural de América Latina en la actualidad. En la segunda, los obispos tratan de juzgar esa realidad a la luz de la fe, y en la tercera articulan las prioridades pastorales en el futuro, que constituirán la respuesta de la Iglesia ante los desafíos contemporáneos.

En la descripción y evaluación de los obispos de la América contemporánea hay una serie de observaciones interesantes. En primer lugar, el catolicismo ya no se describe no como un elemento central en la cultura latinoamericana como lo hacían las conferencias pasadas (véase por ejemplo CELAM DP, 1979: \#412). En el documento de Aparecida, el catolicismo se plantea como una realidad histórica-cultural en América 
Latina y como una herencia preciosa que se podría perder. Así, la conexión directa entre la cultura y la religión es menos que antes (CELAM DA, 2007: \#8; Verdugo, 2008). En su relato de los cambios sociales, culturales y económicos que caracterizan a América latina, los obispos usan el concepto de la globalización y advierten sobre la polarización económica, los acuerdos

Los comerciales injustos con las empresas multinacionales, la corrupción y la falta de una política con compromiso social en las clases políticas de América Latina (CELAM DA, 2007: \#78). Sin embargo, todo el documento de Aparecida enfoca su descripción en los procesos de cambio cultural que se viven en esta parte del mundo. Aquí la evaluación, en general, es negativa. Según los obispos, América latina se caracteriza por el relativismo moral y religioso y la anomalía existencial: “Vivimos un cambio de época, cuyo nivel más profundo es el cultural. Se desvanece la concepción integral del ser humano, su relación con el mundo y con Dios [...] Surge hoy, con gran fuerza, una sobrevaloración de la subjetividad individual" (CELAM DA, 2007: \#44). Los obispos calculan que el catolicismo popular y tradicional no es un contrapeso suficiente para el relativismo generalizado. Por lo tanto, la Iglesia debe buscar a quienes son católicos por costumbre:

No resistiría a los embates del tiempo una fe católica reducida a bagaje, a elenco de algunas normas y prohibiciones, a prácticas de devoción fragmentadas, a adhesiones selectivas y parciales de las verdades de la fe, a una participación ocasional en algunos sacramentos, a la repetición de principios doctrinales, a moralismos blandos o crispados que no convierten la vida de los bautizados (CELAM DA, 2007: \#12).

Con el fin de cambiar lo mencionado líneas atrás y de renovar la fe, los obispos lanzan una "nueva misión continental" en la tercera parte del documento; una campaña que la Iglesia idealmente debe dirigir a todos los sectores y grupos sociales de la sociedad. Esta tiene como objetivo hacer de todos los bautizados "discípulos y misioneros" a través de un "encuentro personal con Jesucristo", que se describe como "una experiencia religiosa profunda e intensa [...] que lleve a una conversión personal y a un cambio de vida integral" (CELAM DA, 2007: \#226). Cada diócesis debe hacer un plan pastoral que redefina la estrategia pastoral de "conservación" a "misión". Al mismo tiempo, el documento solicita a todas las parroquias que salgan de las iglesias para realizar una misión en las calles (CELAM DA 2007: \#154-156; 226).

Si se revisa cuidadosamente dicho documento, se puede ver que hay 140 referencias a la palabra "misión", 149 a "Espíritu”, 47 a "una encuentro (personal) con Jesús", 46 a "conversión", 44 a "experiencia de Dios/Jesús" y 73 referencias a "alegría" (CELAM DA, 2007; Thorsen, 2015). Tanto el lenguaje como las propuestas pastorales del documento Aparecida tienen un tono de avivamiento y misión sorprendente, en especial si se compara con los documentos anteriores del CELAM. 
Este enfoque en la conversión personal, la experiencia de Jesús y el Espíritu Santo llama la atención en un contexto católico, donde a menudo se enfatiza la tradición y la continuidad. Esto ha llevado a muchos observadores a señalar la influencia de la RCC en la reunión de Aparecida.

El teólogo alemán-brasileño Paulo Suess caracteriza a "las manifestaciones baratas de alegría" en la descripción de la realidad latinoamericana como un signo de la presencia de sectores carismáticos en la conferencia de Aparecida, y plantea el uso constante de la palabra "alegría" como "sobrecalentado", especialmente cuando se "toma en cuenta el hecho de que la palabra cruz no aparece ni una sola vez [en el índice]" (Suess, 2008: 72). La influencia de la RCC también ha sido demostrada y descrita por otros teólogos (véase, por ejemplo Arntz, 2008; Libânio, 2008). El entonces director del Centro Bíblico Pastoral para América Latina del CELAM (2004-2011), Fidel Oñoro Consuegra CJM, afirmó que en Aparecida los obispos habían empleado el mismo lenguaje que el de la Renovación Carismática, ya que "la renovación de una manera significativa ha logrado penetrar el nivel institucional de la Iglesia" (comunicación personal, 19 de marzo del 2011).

\section{La Misión Continental en Guatemala}

La RCC ha jugado un papel vital en la recepción del Documento Aparecida (DA) en la Iglesia católica de Guatemala; en especial a partir de su aplicación en el "Plan Global 2008-2016" de la Conferencia Episcopal de Guatemala (CEG), en una "Fiesta Misionera" de la Arquidiócesis de Guatemala en agosto 2009 y en el Congreso Misionero de Guatemala (COMGUA 3) realizado en noviembre del mismo año.

Recuérdese que el episcopado guatemalteco desde los principios de los años setenta ha visto a la RCC con precaución. El primero de estos es un plan pastoral propuestos para el periodo 2008-2016; se estructura siguiendo el modelo de ver-juzgaractuar y se inicia con un análisis de la realidad eclesial contemporánea de Guatemala. Los obispos guatemaltecos alaban la pluralidad de la participación de los laicos en la Iglesia, pero también subrayan la necesidad urgente de un mejor control eclesial con muchos de los llamados "predicadores", quienes hacen un tratamiento de la Biblia que "está inmerso en [un] tipo de interpretación de la Palabra de Dios" caracterizado por el "fundamentalismo" (CEG, 2008: 20). Los obispos igualmente critican el hecho de que muchos dedicados a la santificación personal no están dispuestos a comprometerse con la doctrina social de la Iglesia y la transformación de la realidad temporal (\#26). Por su parte, los medios de comunicación católicos privados se les critica por "transmitir informaciones, preferentemente culturales, orientaciones doctrinales muy tradicionales, desencarnadas y teóricas y muy poco preocupados por «crear conciencia» madura y responsable frente a los graves problemas que afectan a nuestra sociedad y a nuestros pueblos” (\#27). Aunque los obispos no mencionan que la RCC y las 
radios nacionales tienen vínculos con el movimiento carismático (por ejemplo Radio Estrella, Radio Eventos Católicos y Radio Católica Hosanna), resulta obvio que este es el objetivo de las advertencias. El Plan Global continúa con un análisis de la sociedad guatemalteca contemporánea, tanto en las dimensiones socioculturales, como en las económicas y políticas. De eso se preocupan los primeros cuatro capítulos analíticos (que corresponden al "ver" y "juzgar").

La parte analítica sigue el contorno del DA, pero dedica más tiempo al análisis de la situación política y económica de Guatemala, pues presenta una dura crítica a la distribución de ingresos y riquezas en el país, a la pobreza masiva endémica y a la corrupción de la élite política y financiera. Luego, los obispos se dedican a lo que ellos ven como la misión pastoral de la Iglesia en la sociedad guatemalteca, a partir del DA:

Trabajar para que la Iglesia en Guatemala se renueve en sus miembros y en sus estructuras a partir del encuentro con Jesús, para ser sus discípulos y misioneros y testigos del Reino de Dios en la realidad actual de Guatemala, de modo que nuestro pueblo tenga en Jesucristo la vida, la esperanza y la alegría que vienen de Dios (CEG, 2008: 53).

Todas las palabras claves de Aparecida se contemplan en este "objetivo general"; sin embargo, el lenguaje de renovación y avivamiento se combina directamente con un enfoque a la justicia social, visible por ejemplo en las palabras de los "testigos del Reino". Parece como si los obispos guatemaltecos en su adaptación del DA estuvieron ansiosos de no dejar la interpretación del DA en manos de los sectores carismáticos de la Iglesia y sus medios de comunicación. Por este motivo, en repetidas ocasiones hacen una clara conexión entre la conversión personal y el compromiso social:

A quienes hemos sido vivificados por la vida de Cristo nos toca manifestar el inmenso amor del Padre que quiere que todos seamos hijos suyos. De todos los que hemos acogido la vida en Cristo, se espera un testimonio muy creible, comprometido y valiente de los tesoros de gracia y de humanización que la vida de Dios comporta para todos sus hijos e hijas [...] Desde esa convicción, queremos reiterar la intima relación entre Reino de Dios, justicia social y caridad cristiana, subrayando la dimensión transformadora del Reino (CEG, 2008: \#50-51).

El hecho de que los sectores laicos carismáticos estén ansiosos por interpretar e implementar la Misión Continental del DA se demuestra en los siguientes dos eventos. En noviembre del 2009, la Iglesia católica en Guatemala se preparaba para el tercer congreso misionero (COMGUA), con cual la Misión Continental lanzada en Aparecida se inició oficialmente en el país. El COMGUA 3 se celebró en la ciudad caribeña Puerto Barrios del 13 al 15 de noviembre, y fue el evento donde los obispos, durante la misa final del domingo, enviaron formalmente a los dos mil delegados para iniciar la fase activa de la Misión Continental en Guatemala. Una semana antes, la Arquidiócesis 
de la Ciudad de Guatemala celebró una fiesta misionera en la preparación local del COMGUA 3. Se llevó a cabo en el enorme auditorio "Juan Pablo II", la sede nacional de la RCC (Calzada Roosevelt, Mixco, Dept. de Guatemala). Ambos eran eventos masivos caracterizados por una atmósfera casi eufórica por motivo de la Misión Continental. Sin embargo, también había ciertas diferencias en la manera en que el esfuerzo misionero se entendía y se celebraba. Quizás estas discrepancias pueden atribuirse al grado de influencia de los sectores carismáticos en las diferentes partes de la Iglesia.

La fiesta misionera de la capital fue organizada por el Consejo Arquidiocesano de los Laicos y terminó con una misa festiva celebrada por el obispo auxiliar Rodolfo Mendoza. El evento mantuvo un estilo semicarismático, tanto en la predicación laica, como en las alabanzas y las oraciones fervientes. Los participantes parecían ser miembros de los cientos de grupos de oración del área metropolitana.

El COMGUA 3, además, fue organizado por el Vicariato Apostólico de Izabal y su entonces obispo Mons. Gabriel Peñate, en coordinación con la CEG. El congreso tuvo lugar en el polideportivo "Sto. Tomás de Castilla" en Puerto Barrios, y participaron delegaciones de sacerdotes, religiosas, laicos y jóvenes de todo el país. El evento comenzó con una procesión de entrada llevando enormes fotos de los mártires, sacerdotes y catequistas del vicariato, asesinados por grupos paramilitares durante el pico del conflicto armado interno a inicios de la década de los ochenta. Las imágenes fueron colocadas en frente del escenario y permanecieron en la sala durante todo el evento. La música, las canciones y los himnos eran muy diferentes a las alabanzas de los grupos carismáticos. Aparte de un himno compuesto especialmente para el COMGUA 3, se entonaron las canciones de los años 1970 y 1980; todos sobre la Iglesia, la esperanza, la justicia, el Reino de Dios y el Pueblo de Dios. Se sentía el toque de la Acción Católica, la teología de la liberación y la experiencia de persecución.

Los diferentes ponentes trataron el tema de la "misión y evangelización" desde diferentes perspectivas. Entre ellos, el padre Víctor Ruano de la diócesis de Jalapa dio un discurso alentando sobre la participación eclesiástica en el combate contra las injusticias. Ruano criticó a la élite económica de Guatemala y las corporaciones mineras internacionales con tanta fuerza que algunos de los periodistas presentes dijeron, en tono de broma, que era necesario conseguir buenas fotografías del Padre Ruano antes de que lo mataran. En el congreso COMGUA se vivía una atmósfera mezclada, por un lado, entusiasta con gritos de júbilo y porras carismáticas; por otro lado, seria en los discursos, los talleres y en los himnos y canciones propios del ámbito eclesial de la Acción Católica.

Ante esto, al menos el 75 por ciento de los participantes laicos provenían de los grupos laicos y movimientos carismáticos. Al comparar el COMGUA 3 con los congresos misioneros anteriores, el Hrno. Santiago Otero FMS, sociólogo y exsecretario de la Conferencia Episcopal de Guatemala, se puede caracterizar a este tercer congreso como un momento donde el grupo carismático entre los laicos ponía su marca distintiva 
en el evento (Diario de campo, 18 de noviembre de 2009). En suma, el COMGUA 3 se caracterizó por la misma alegría festiva como en eventos carismáticos a pesar de que el marco del congreso era no-carismático (discursos, oraciones y música). De esa manera, se demostró la diferencia entre laicos e Iglesia oficial, y entre los carismáticos y los de la Acción Católica. El congreso finalizó con una misa, en la cual los obispos enviaron formalmente a los participantes a sus diócesis para comenzar la Misión Continental.

\section{La Misión Continental a nivel parroquial}

Durante el otoño del 2009, en diferentes grupos de oración en la parroquia de la Santísima Trinidad y en los eventos y grupos en la Arquidiócesis de Guatemala, la "Misión Continental" era un tema frecuente en la predicación laica. En octubre de ese mismo año se informó que el Consejo Parroquial de la Santísima Trinidad había decidido organizar una misión en la colonia residencial, la cual se pondría en marcha en noviembre e incluiría a todos los grupos de laicos. A continuación se explorará cómo se recibió la DA en el grupo de oración del Espíritu de Dios y cómo se realizó la campaña de misión local en la parroquia.

En el grupo del Espíritu de Dios, doña Concha había decidido dedicar las cuatro reuniones del mes de noviembre al estudio del documento de Aparecida y de la Misión Continental. Ella destinaba una parte de la sesión para conversar sobre los diferentes temas del DA, y los predicadores laicos invitados también habían sido instruidos para hablar sobre la misión. La base de las charlas y sermones era una libreta, que la Arquidiócesis de Guatemala había publicado poco después del "Plan Global 2008-2016", mencionado anteriormente. La libreta se titulaba "20 Mensajes de Aparecida", y se utilizaría en la formación de los agentes pastorales -sacerdotes, religiosas y laicos comprometidos- para la Misión Continental (Arq. Guatemala, 2009). Sin embargo, doña Concha los usaba para animar al grupo del Espíritu de Dios, ya que para ella "todos somos pequeños agentes pastorales" (Diario de campo, 4 de noviembre del 2009). Con la libreta en la mano, doña Concha agitó:

¿Saben qué, hermanos? La Iglesia de hoy se está poniendo estricta, fuerte y exigente. Exige de nosotros que profundicemos nuestro conocimiento de la doctrina de la Iglesia y que profundicemos nuestro compromiso. ¿Y saben por qué? Porque las iglesias protestantes, las sectas, están llenas de católicos y eso no debe seguir así. [...] Nuestros obispos quieren que salgamos de nuestros templos para anunciar el Evangelio en las calles. La Iglesia necesita gente de fe, porque los que tienen fe, tienen a Dios adentro, y sabemos que él puede hacer cosas increíbles [...]. (Diario de campo, 4 de noviembre del 2009).

Después de algunas alabanzas, doña Concha introdujo al predicador (laico) de esa sesión, quien dedicaría su sermón al primer ejercicio de la libreta, que consistía en una introducción a los 18 primeros párrafos del documento Aparecida con preguntas 
de reflexión sobre el contenido. Dado un mal entendido, el predicador pensó que el título "Números 1-18" (de la DA) era una referencia bíblica, y comenzó leyendo el capítulo uno, versículo dieciocho del Libro de los Números, que trata de la numeración de los israelitas en el desierto. Después de un momento de silencio pensativo, el predicador razonó que al igual que Dios había contado a todos los israelitas, los obispos habían decidido que toda la gente debería ser misionera con el fin de llevar la palabra de Dios a todas partes. Continuó diciendo que la razón de la "enfermedad en la Iglesia" eran "los católicos superficiales", quienes se llamaban a sí mismos católicos, pero en realidad eran "espiritualmente muertos", pues "no conocen a Jesús". Luego se volvió hacia la libreta y habló sobre las tres palabras clave del primer ejercicio: "Discipulado", “Misión" y "La vida (vertical y horizontal)" (Arq. Guatemala, 2009: 3). Al igual que doña Concha, explicó la necesidad de la misión a la luz de las iglesias protestantes y terminó exclamando: “Si no nos preparamos ahora, los protestantes nos van a seguir persiguiendo así", y abría y cerraba la mano tres veces imitando un cocodrilo (Diario de campo, 4 de noviembre del 2009).

El miércoles siguiente, me había preparado leyendo cuidadosamente la libreta con el fin de ver cómo el próximo predicador aplicaba el texto en su sermón. El segundo ejercicio fue sobre el método ver-juzgar-actuar, y el predicador comenzó leyendo cuidadosamente en voz alta las cinco primeras líneas del documento:

I. Metodología del Documento: Ver/juzgar/actuar. Ver: Para mirar la realidad con ojos de creyentes... Pero para mirarla. No podemos hacer pastoral en la iglesia "sobrevolando" por encima de la realidad que nos rodea. Para juzgarla a la luz del Evangelio y de la enseñanza de la iglesia. TY para actuar proyectando las acciones que debemos emprender para transformarla. (Arq. Guatemala, 2009: 5).

Después de estas palabras, el predicador se separó de la lectura del texto y dijo: "Hay cinco pasos: ver, juzgar, actuar, evaluar y celebrar", por lo cual añadió las dos últimas que no se encontraban en el texto de la libreta. El hecho de que el predicador utilizara esa versión en lugar de la del libro indica que es el uso común en círculos carismáticos, lo cual no puede ser ninguna sorpresa, pues la dimensión festiva y la celebración de la fe es un elemento central. El predicador luego invitó a los presentes a unirse a una examinación de "las cosas que nos rodean", y con el fin de explicar el método eligió a "nuestras familias" y a "nuestro grupo de oración" como ejemplos. El predicador utilizó el primer ejemplo de "la familia", y pidió a los asistentes imaginarse una "familia con divisiones". Describió "la falta de respeto entre los cónyuges y entre padres e hijos", "el alcoholismo" y "la desintegración". Además, solicitó juzgar la situación a la luz de la fe, hacer un diagnóstico de los males e indicar las causas. Él, por su parte, nombró varios motivos, entre ellos la falta de moral, de respeto, de educación de los hijos, etc., que finalmente se podían resumir en una "falta de fe en Dios". 
¿Qué hacer?, preguntaba retóricamente; quizás invitar a la familia para ir a un grupo de oración o tal vez a un retiro para parejas, donde puedan reconciliarse y aprender a perdonarse, propuso el predicador. Luego se debe comenzar a ir a misa juntos y a asistir a un grupo. Después de un tiempo la familia debe evaluar su situación: qué ha cambiado, qué ha mejorado, pero aún necesita corrección. Por último, la familia y la comunidad deben dar gracias a Dios y celebrar el hecho de que él tiene el poder para "cambiar las cosas" y "sanar a las familias" (Diario de campo, 11 de noviembre de 2009). Luego de la familia, el predicador tomó el ejemplo de un grupo de oración que había perdido el fervor de evangelizar. Aunque él utilizaba muchos de los términos de la libreta, se hizo evidente que aplicó el material de una forma muy diferente a la prevista por los autores en la Comisión Arquidiocesana para la Misión Continental. En la libreta, el método ver-juzgar-actuar se destina a analizar la situación política, social y eclesial en la sociedad, en cambio aquí se utilizaba como una herramienta para estudiar la moralidad de la familia y la disposición misionera del grupo de oración carismático.

Sin embargo, la libreta de la Comisión Arquidiocesana fue compuesta por dos sacerdotes, Carlos Martínez y Pedro Jaramillo, la hermana religiosa Raquel Saravia y dos predicadores laicos carismáticos, Juan Ramón Martínez y Byron Valdizón. La libreta consiste de 20 ejercicios o lecciones destinados a trabajos en grupo para los agentes pastorales siguiendo el método "ver-juzgar-actuar" (Arq. Guatemala, 2009: 5). Aunque habrán dos miembros carismáticos, parece como si los miembros religiosos tuvieron la mayor influencia en la interpretación del DA en la libreta. En comparación con el original, esta versión es mucho más "suave" en su retórica; pues palabras como "Espíritu”, "encuentro personal”, "alegría” y "conversión” se reproducen con menor densidad y se conectan inmediatamente con temas como la justicia social, la dignidad humana y la opción preferencial por los pobres. En la lucha por la interpretación del DA, el p. Pedro Jaramillo ha hecho hincapié en la continuidad del DA con las conferencias anteriores del CELAM y la "opción preferencial por los pobres". En su libro titulado La espiritualidad del discípulo misionero [de Aparecida] (2008), Jaramillo advierte contra una lectura espiritual "intimista e individualista" del DA, y por tanto interpreta el documento como uno donde se entrelazan la espiritualidad y el compromiso social. Esto lo hace para calmar a quienes tienen miedo de que el DA "podría ascender a las nubes" de un "espiritualismo estéril" (Jaramillo, 2008: 13). En la libreta de la arquidiócesis, Jaramillo y los otros autores parecen haber tratado de garantizar que las dimensiones humanas "horizontales" del DA no se descuidan en favor de las dimensiones "verticales" y estrictamente espirituales.

De este modo, el análisis del uso de la libreta en el grupo de oración del Espíritu Santo es ilustrativo de los diferentes énfasis que se les dan a los elementos centrales de la DA en las interpretaciones del documento por la iglesia institucional y por los grupos laicales carismáticos. En la libreta, el compromiso de la Iglesia por la justicia 
social es visto como parte integral de la Misión Continental, mientras que los grupos carismáticos -a nivel parroquial, diocesano y nacional- están más enfocados en una agenda estrictamente espiritual, la cual tiene como principal objetivo promover conversiones personales para cambiar el país, es decir, el cambio personal fortalece a la Iglesia católica ante el proselitismo de las iglesias evangélicas.

La semana de misión de la parroquia en noviembre estaría seguida por una campaña de "puerta a puerta" en diciembre del 2009. Aquí voluntarios de todos los grupos de laicos (entre ellos también los ministros extraordinarios de la comunión y los lectores) planeaban ir de a todas las casas del área para hablarle a la gente acerca de la fe católica, informar sobre la parroquia, invitarles a ir a misa y a participar en algún grupo de oración. Además iban a preguntar si los niños estaban bautizados e informar sobre cursos prebautismales y prematrimoniales. La parroquia ya había organizado una campaña similar cinco años ante s, y según doña Concha el consejo parroquial había decidido llevar a cabo ese tipo de actividad cada dos años. Al respecto, doña Mimi, una servidora de otro grupo carismático, quien había participado en la campaña anterior, comentó.

¿Sabe qué?, la gente nos recibe muy bien. ¡Por supuesto que los protestantes quieren discutir!, pero la gente que es católica nos recibe muy bien. A veces cuando tocamos la puerta, piensan que somos mormones o testigos de Jehová, y gritan: "No, gracias, somos católicos" desde el interior de la casa. Pero cuando les decimos que venimos de la parroquia, nos reciben bien. Hay mucha genta allá abajo [señalando hacia la zona más pobre de la vecindad] que son recién llegados y alquilan las casas allá. Ellos no saben bien dónde queda la iglesia y lo que pasa en la iglesia. Así que les decimos todo y les preguntamos que si quieren que se bauticen sus bebés (comunicación personal, 5 de agosto de 2009).

En sus sermones, el párroco habló muy positivo de la iniciativa de la semana de misión, pero él no participó, ni ayudó con la organización de las reuniones al aire libre, la campaña de puerta a puerta o el retiro final. Todo estaba en manos del consejo parroquial, formado por representantes de los diferentes grupos de laicos, de los cuales la mayoría eran carismáticos. Aunque sea diferente en otras parroquias se ve la tendencia de que la iniciativa misional esté en manos de laicos avivados como en "la fiesta misionera" o el congreso misionero (COMGUA 3).

Ya en 1992, Edward Cleary señaló que los nuevos movimientos católicos "imitan a las iglesias no católicas" (Cleary, 1992: 215), y en 1994 Phillip Berryman escribió que "la renovación carismática católica, 'Trigo' [ministerio de predicación laica en la Cuidad de Guatemala] y SINE tienen una serie de características similares a las iglesias evangélicas" (Berryman, 1994: 168). Por ejemplo el tipo de misión de puerta a puerta era hasta hace poco una característica de las iglesias evangélicas, de los mormones y de los Testigos de Jehová. Al respecto, Cleary (1992) describió cómo una campaña de 
misión de puerta a puerta en una parroquia de la Ciudad de Guatemala en 1989 tenía gran éxito en atraer a conversos a las iglesias evangélicas para que regresaran al catolicismo. En ese momento, el método era controversial, y la mayoría de los párrocos eran reacios a utilizarlo. Sin embargo, las circunstancias están cambiando, pues en el año 2009, las misiones de puerta a puerta se habían tornado mucho más frecuentes en las parroquias de Guatemala, y con la Misión Continental esta tendencia probablemente se reforzará aún más. Así, católicos carismáticos toman la iniciativa en la aplicación de las nuevas técnicas de la misión.

La predicación laica en la calle al aire libre es también algo nuevo en un contexto católico. Predicadores de la calle eran hasta hace poco un fenómeno exclusivamente evangélico y la Iglesia católica siempre huturo los obispos demuestran su confianza en una iglesia de "misioneros y discípulos" que han experimentado un "encuentro personal con Jesucristo" y que son serenos en su fe, activos y comprometidos. Además se destaca un cierto menosprecio por el catolicismo popular y cultural. Durante el periodo de investigación en Ciudad de Guatemala, varias veces oí las quejas de católicos no-carismáticos de las campañas de evangelización, entre ellas las de una vecina que me decía: "Hay demasiado aleluya en la iglesia hoy en día". La agenda eclesial de la "misión continental" tiene como consecuencia que el impacto de los sectores carismáticos es más grande y las nuevas empeñas misionales. Con la RCC y el proyecto eclesial de la nueva evangelización, la Iglesia católica de Guatemala y Latinoamérica adquiere un perfil más parecido a las iglesias evangélicas; es decir más estrechamente denominacional.

\section{¿"Pentecostalización” de la Iglesia católica?}

Comoseha planteadoen este artículo, hay un fuerteimpactodela RCC en la Iglesia católica en Guatemala y en Latinoamérica. Este movimiento predominantemente laico influyó tanto a nivel institucional como parroquial. Para la Iglesia, el entusiasmo y compromiso es un gran activo en el proceso de la llamada "nueva evangelización". Es obvio para los observadores del campo religioso en Latinoamérica que la Iglesia católica está viviendo un periodo de avivamiento, donde el laicado se ha vuelto mucho más activo y la Iglesia ya no depende tanto de personal y de fondos económicos de Europa y EE. UU. Además, los seminarios están más llenos que nunca y se vive una atmósfera de esperanza y entusiasmo (Cleary, 2009; Thorsen 2015). Parte del avivamiento es debe a la adopción de varios elementos de la tradición pentecostal que han entrado al catolicismo mediante la RCC, por ejemplo, en los grupos de laicos se cantan las alabanzas evangélicas, se aplaude, se da testimonio, hay predicación bíblica, se ora por los enfermos, se exorcizan los demonios y se habla en lenguas. El porcentaje de católicos en Guatemala que ha practicado uno de los elementos mencionados es considerable. Al mismo tiempo, la Iglesia en Latinoamérica y en Guatemala ha iniciado otra etapa de 
la "nueva evangelización" (la "misión continental"), que parece adoptar un lenguaje y una línea pastoral semejantes a las iglesia evangélicas y pentecostales.

Ante esto, vale la pena preguntarse si también la Iglesia católica en Latinoamérica (y Guatemala) está viviendo un proceso de "pentecostalización”, como lo han sugerido varios académicos (Chesnut, 2003 y 2016; Espinosa, 2004; Gooren, 2010). Pese a esta idea, se podría tratar el tema con más cautela y proponer, más bien, una "incipiente pentecostalización" del catolicismo guatemalteco y latinoamericano (Thorsen, 2015). La razón de esta cautela es que para hablar de una pentecostalización completa de la Iglesia también se debe demostrar que ha penetrado el nivel institucional. Como vimos, hay indicaciones de eso en el documento Aparecida y en el "Plan Global" de la Conferencia Episcopal de Guatemala (CELAM DA, 2007 y CEG, 2008).

Sin embargo, también se puede argumentar que eso "solo" es una continuación del programa de la nueva evangelización envuelto en un lenguaje de tinte carismático. Pero eso representa un gran cambio y un nuevo rol de la religión católica, según el cual la Iglesia cada vez parece más una denominación y cada vez menos un marco religioso-cultural universal. Los obispos de Latinoamérica y Guatemala han optado más por una Iglesia misionera, confesional y comprometida; lo cual corresponde a las prioridades de la RCC y, por lo tanto, el celo misionero de la cúpula y de la base de la iglesia se están uniendo en el intento de alcanzar las masas católicas.

En el Documento Aparecida, los obispos describen la fe popular como "fragmentada" e "incapaz de resistir los desafíos y cambios del tiempo" (CELAM DA, 2007: \#12). Por lo tanto, para el futuro los obispos demuestran su confianza en una iglesia de "misioneros y discípulos" que han experimentado un "encuentro personal con Jesucristo" y que son serenos en su fe, activos y comprometidos. Además se destaca un cierto menosprecio por el catolicismo popular y cultural. Durante el periodo de investigación en Ciudad de Guatemala, varias veces oí las quejas de católicos no-carismáticos de las campañas de evangelización, entre ellas las de una vecina que me decía: "Hay demasiado aleluya en la iglesia hoy en día". La agenda eclesial de la "misión continental" tiene como consecuencia que el impacto de los sectores carismáticos es más grande en la Iglesia católica de Guatemala, pues para la realización de los planes pastorales se cuenta con la participación activa del laicado.

\section{Notas}

1 El trabajo de campo se llevó a cabo en una parroquia de una colonia residencial de la Ciudad de Guatemala. Perfil socioeconómico: clase media-baja. El autor asistió a dos diferentes grupos carismáticos en la colonia, más uno juvenil, carismático no parroquial que se juntaba en un hotel elegante en una zona afluente de la misma capital. Aparte de eso el autor asistió a varios mega eventos carismáticos, visitó varios comunidades y ministerios carismáticos. Además participó como observador en el tercer Congreso de Guatemala (COMGUA3) que se llevó a cabo del 13 al 15 de noviembre 2009 en Puerto Barrios, Izabal. En total se condujeron 35 entrevistas: 8 con líderes y predicadores carismáticos, 10 con sacerdotes y reliogisos, 13 con participantes de grupos carismáticos y 2 con los obispos Mons. 
Gustavo Rodolfo Mendoza Hernández y Mons. José Ramiro Pellecer Samayoa. El trabajo de campo está descrito en detalle en el libro: Carismatic Practice and Catholic Parish Life - The Incipient Pentecostalization of the Church in Guatemala and Latin America (Thorsen, 2015). Este artículo también se basa en material de un trabajo de campo (de enero a mayo del 2005) anterior en la Ciudad de Guatemala, más un trabajo de campo llevado a cabo de octubre del 2014 a enero del 2015 en Santiago Atitlán, Sololá, Guatemala. noviembre de 2009).

Como se indicó arriba, el trabajo de campo se llevó a cabo de mayo a diciembre del año 2009. Los nombres de la parroquia, de la colonia residencial, de los grupos de oración y de los informantes utilizados en este trabajo son ficticios, con el fin de velar por el anonimato de las personas participantes.

\section{Entrevistas y fuentes}

Mendoza Hernández, Monseñor Gustavo Rodolfo. Entrevista. Entrevistador: Jakob Egeris Thorsen. Ciudad de Guatemala, 5 de noviembre de 2009 [no publicada].

"Mimi", servidora en la "Parroquia Santísima Trinidad". Entrevista. Entrevistador: Jakob Egeris Thorsen, Ciudad de Guatemala, 5 de agosto de 2009 [no publicada].

Oñoro, Fidel CJM, Entrevista. Entrevistador: Jakob Egeris Thorsen. Sacrofano, Roma, 19 de marzo 2011.

Pellecer Samayoa, Monseñor José Ramiro. Entrevista. Entrevistador: Jakob Egeris Thorsen. Sacatepéquez, 27 de noviembre de 2009 [no publicada].

Thorsen, Jakob Egeris. Diario de campo 2009. Ciudad de Guatemala [no publicado].

\section{Bibliografía}

Althoff, Andrea. Religion im Wandel: Einflüsse von Ethnizität auf die religiöse Ordnung am Beispiel Guatemalas. Halle-Wittenberg: Martin-Luther Universität Halle-Wittenberg, PhD Dissertation, 2005.

Arntz, Norbert. "Einführung in Aufbau und Inhalt des Schlussdokumentes von Aparecida". Zeitschrift für Missionswissenschaft un Religionswissenschaft. Jg.92, H. 1, 2008.

Arq.Guatemala. Arquidiócesis de Guatemala: Misión Continental, 20 Mensajes de Aparecida. Etapa: Sensibilización de Agentes de Pastoral. Por la Cominsión Arquidiocesana para la Misión Continental. Ciudad de Guatemala: Arquidiósesis de Guatemala, 2009.

Barrett, David B., y Co. World Christian Trends, AD 30-AD 2200: Interpreting the Annual Christian Megacensus. Pasadena, California: William Carey Library, 2001.

Berryman, Phillip. Stubborn Hope: Religion, Politics, and Revolution in Central America. Maryknoll, New York: Orbis Books, 1994.

CCR Guatemala. Censo Nacional de la Renovación Carismática Católica en Guatemala 2008. Ciudad de Guatemala: no publicado.

CELAM DA. V Conferencia General del Episcopado Latinoamericano y del Caribe. Documento Conclusivo. Bogotá: San Pablo, 2007.

CELAM SD. IV Conferencia Episcopal Latinoamericana - Documento de Santo Domingo. Bogotá: CELAM, 1992 
CELAM DP. Tercera Conferencia General del Episcopado Latinoamericano. Documento de Puebla. Bogotá: CELAM, 1979.

CEG. Conferencia Episcopal de Guatemala: Renovados en el Espiritu - Instrucción Pastoral Colectiva de los Obispos de Guatemala sobre la Renovación Carismática. Ciudad de Guatemala, CEG, 1986.

CEG. Conferencia Episcopal de Guatemala - Plan Global 2008-2016. Ciudad de Guatemala: CEG, 2008.

Chesnut, Robert Andrew. Competitive Spirits. Latin America's New Religious Economy. Oxford \& New York: Oxford University Press, 2003.

Chesnut, Robert Andrew. "Latin American Charisma: The Pentecostalization of Christianity in the Region". New Ways of Being Pentecostal in Latin America. Martin Lindhardt (Ed.). London: Lexington Books, 2016. 1-14.

Cleary, Edward L. "Evangelicals and Competition in Guatemala". Conflict and Competition: The Latin American Church in a Changing Environment. Eds. Edward Cleary y Hanna Steward-Gambino. Boulder, Colorado: Lynne Reinner Publishers, 1992.

Cleary, Edward L. "Latin American Pentecostalism". The Globalization of Pentecostalism. Eds. Murray W. Dempster y Co. Oxford: Regnum Books, 1999.

Cleary, Edward L. "Shopping Around: Questions about Latin American Conversion". International Bulletin of Missionary Research, 28(2) (2004): 50-54.

Cleary, Edward L. "The Catholic Charismatic Renewal: Revitalization Movements and Conversion". Conversion of a Continent: Contemporary Religious Change in Latin America. Eds. Timothy J. Steigenga y Edward L. Cleary. New Brunswick \& London: Rutgers University Press, 2007.

Cleary, Edward L. How Latin America Saved the Soul of the Catholic Church. Mahwah, New Jersey: Paulist Press, 2009.

Cleary, Edward L. The Rise of Charismatic Catholicism in Latin America. Florida: University Press of Florida, 2011.

Early, John D. Maya and Catholic Cultures in Crisis. Florida: University Press of Florida, 2012.

Espinosa, Gaston. "The Pentecostalization of Latin American and U.S. Latino Christianity". Pneuma, 26, 2 (2004): 262-292.

Falla, Ricardo. Quiché Rebelde. Religious Conversion, Politics, and ethnic Identity in Guatemala. Austin: University of Texas Press, 2001.

Gooren, Henri. "The Pentecostalization of Religion and Society in Latin America”. Exchange, 39, 4 (2010): 355-376.

Hoenes del Pinal, Eric. "Towards an Ideology of Gesture: Gestures, Body Movement and Language Ideology among Q'eqchi'-Maya Catholics." Beyond Logos: Extensions of the Language Ideology Paradigm in the Study of Global Christianity(-ies). Special Section of Anthropological Quarterly 84, 3 (2011): 595-630.

Jaramillo Rivas, Pedro. Espiritualidad del Discípulo Misionero - 100 pistas del camino de Aparecida. México, D.F.: Desclée De Brouwer, 2008.

Libanio, João B. "Conferencia de Aparecida. Documento final". Revista Iberoamericana De Teología, 2008: 23-46.

MacNutt, Francis. Healing. Notre Dame, Indiana: Ave Maria Press, 1974.

MacNutt, Francis. Deliverance from Evil Spirits: A Practical Guide. Grand Rapids, Michigan: Baker Books, 1995. 
Melander, Veronica. The Hour of God? People in Guatemala Confronting Political Evangelicalism and Counterinsurgency (1976-1990). Uppsala: Acta Universitatis Upsaliensis, 1999.

O’Neill, Kevin Lewis. City of God. Christian Citizenship in Postwar Guatemala. Berkeley: University of California Press, 2010.

Pew Forum on Religion \& Public Life. Spirit and Power: A 10-Country Survey of Pentecostals. Washington: Pew Research Center, 2006.

Pew Forum on Religion \& Public Life. Religion in Latin America._Washington: Pew Research Center, 2014.

Steigenga, Timothy. J. The Politics of the Spirit: The Political Implications of Pentecostalized Religion in Costa Rica and Guatemala. Maryland: Lexington Books, 2001.

Stoll, David. Between Two Armies in the Ixil Towns of Guatemala. New York: Columbia University Press, 1993.

Suess, Paulo. "Die missionarische Synthese des Schlussdokuments von Aparecida". Zeitschrift für Missionswissenschaft und Religionswissenschaft. Jg.92, H. 1. (2008).

de Theije, Marjo. "Charismatic Renewal and base Communities: The Religious Participation of Women in a Brazilian Parish". More Than Opium: An Anthropological Approach to Latin American and Caribbean Pentecostal Praxis. Eds. Barbara Boudewijnse, A. F. Droogers, Frans Kamsteeg. Lanham: Scarecrow Press, 1998. 225-248.

Thorsen, Jakob Egeris. Charismatic Practice and Catholic Parish Life. The Incipient Pentecostalization of the Church in Guatemala and Latin America. Leiden: Brill, 2015

Verdugo, Fernando. "Aparecida: Perspectiva teológico-cultural". Teología y Vida, 49, 4 (2008): 673-684.

Jakob Egeris Thorsen. Danés, bachiller en teología (2003) y antropología social (2005). Licenciatura en teología (2007) de la Universidad de Copenhague. Doctorado en teología (2009-2012) de la Universidad de Aarhus. Profesor asistente en la Sección de Teología, Instituto de Cultura y Sociedad, Universidad de Aarhus, Dinamarca.

Contacto: teojet@cas.au.dk 